\title{
FUNCIÓN PULMONAR EN FALCÉMICOS HOMOCIGOTOS DE 5 A 15 AÑOS EN CONSULTA DE HEMATOLOGÍA, HOSPITAL MATERNO INFANTIL SAN LORENZO DE LOS MINA AGOSTO-OCTUBRE, 2015
}

\author{
Pulmonary function in homozygous phenacecic patients from 5 to 15 years in the hematology \\ practice, Maternal and Child Hospital San Lorenzo de Los Mina August-October, 2015
}

\section{Dra. Claritza Elizabeth Concepción García*, Dra. Rosa Nieves Paulino**, Dra. Elsa Camilo Pantaleón ${ }^{* * *}$, Dr. Rubén Darío Pimentel****}

Recibido: 5/9/2017 Aprobado: 25/10/2017

DOI: http://dx.doi.org/10.22206/cysa.2018.v2i1.pp49-55

\section{Resumen}

Se realizó un estudio descriptivo, retrospectivo, de corte transversal, para determinar la función pulmonar en falcémicos homocigotos de 5 a 15 ańos en la consulta de hematología del Hospital Materno Infantil San Lorenzo de Los Mina, durante el período agosto-octubre de 2015. De un total de 200 pacientes, 23 cumplieron con los criterios de inclusión. La edad más afectada es entre 10-15 años, equivalente a un $57 \%$. El sexo masculino fue el más frecuente, con el 52\%. La neumonía fue la enfermedad pulmonar previa más común, con un $48 \%$. El $30 \%$ de los niños cursó con complicaciones, siendo el Síndrome Torácico Agudo el más reportado, con 6 casos (26\%). Todos los pacientes tenían aplicada la vacuna antineumocócica. El 74\% había sido transfundido. El tratamiento de sostén en el $70 \%$ de los casos era la hidroxiurea, y una combinación de hidroxiurea más terapia transfusional, en el 13\% de los pacientes. Los niveles de hemoglobina más frecuentes correspondieron a la escala de 3-6 gramos y $18-27 \%$, para un total de 11 casos. El patrón ventilatorio más frecuente fue el restrictivo en el $78 \%$ de los pacientes.

Palabras clave: falcémicos homocigotos; función pulmonar; patrón restrictivo; Síndrome torácico agudo.

\footnotetext{
* Pediatra hematóloga del Hospital San Lorenzo de Los Mina, República Dominicana.

** Pediatra hematóloga del Hospital Infantil Dr. Robert Reid Cabral, República Dominicana.
}

\section{Summary}

A descriptive, cross-sectional, retrospective study was performed to determine lung function in homozygous falcemics from 5 to 15 years old at the hematology clinic of the San Lorenzo de Los Mina Hospital during the period August-October 2015. Out of a total of 200 patients, 23 met the inclusion criteria. The most affected age is between 10-15 years, for $57 \%$. The male sex was the most frequent, with $52 \%$. Pneumonia was the most common prior lung disease, with $48 \% .30 \%$ of the children had complications, with acute chest syndrome being the most reported with 6 cases (26\%). All patients had pneumococcal vaccine given. $74 \%$ had been transfused. Supporting treatment in $70 \%$ of cases was hydroxyurea, and a combination of hydroxyurea plus $13 \%$ transfusion therapy. The usual hemoglobin levels corresponded to the scale of 3-6 grams and 18-27\%, for a total of 11 cases. The most frequent ventilatory pattern was restrictive in $78 \%$.

Keywords: homozygous falcemia; pulmonary function; restrictive pattern; Acute thoracic syndrome.

\footnotetext{
*** Pediatra neumóloga del Hospital Infantil Dr. Robert Reid Cabral, República Dominicana. Elsacamilo@hotmail.com

**** Asesor metodológico de la Universidad Autónoma de Santo Domingo.
} 


\section{Claritza Elizabeth Concepción García, Rosa Nieves Paulino, Elsa Camilo Pantaleón y Rubén Darío Pimentel}

\section{Introducción}

La enfermedad de células falciformes o drepanocitosis fue descrita por primera vez en 1910. Se debe a una alteración genética que determina la combinación de hemoglobina (hb) SS y es consecuencia de una mutación de genes que ocurrió hace miles de años en el continente africano; es una hemoglobinopatía que se transmite de forma autosómica recesiva, produciéndose rigidez y deformación del eritrocito en situaciones de hipoxia, infección o deshidratación con la consecuente producción de hemólisis-anemia, hipercoagulabilidad y disfunción endotelial con producción de fenómenos inflamatorios añadidos que generan la consecuente vasculopatía intrínseca y obstrucción vascular, productoras, a su vez, de patologías agudas y crónicas en prácticamente todos los órganos y sistemas del organismo ${ }^{1}$.

La patología respiratoria aguda y crónica ocurre con mucha frecuencia en estos pacientes, constituyendo un factor de riesgo importante para una muerte prematura ${ }^{2}$. Existen diferentes complicaciones a nivel respiratorio, dentro de estas tenemos: Síndrome Torácico Agudo/infección, atelectasias secundarias a crisis de dolor e hipoventilación, enfermedad pulmonar restrictiva, mixta, fibrosis e hipertensión pulmonar, hiperreactividad bronquial, Síndrome de apnea obstructiva de sueño ${ }^{3}$, etc. Por su frecuencia e implicación clínica, se confiere mayor importancia al Síndrome de tórax agudo, que se caracteriza clínicamente por fiebre, dolor torácico, acortamiento de la inspiración, tos y, en los casos graves, hemoptisis, hipoxemia, leucocitosis y disminución relativa de las plaquetas. Una de las particularidades de este síndrome es el inicio súbito y el rápido deterioro de la función pulmonar, lo que amerita intervención y manejo inmediato a fin de evitar mayores complicaciones por esta causa ${ }^{4}$.

El estudio de la función pulmonar es un dato de suma importancia para establecer el pronóstico y la recurrencia de las diferentes complicaciones pulmonares presentadas por los pacientes con anemia falciforme, pero lamentablemente en nuestro país no contamos con estudios al respecto. Lunt et al. ${ }^{5}$ aseguran que las alteraciones de la función pulmonar se producen en niños con enfermedad de células falciformes (ECF) y pueden estar asociadas con el volumen sanguíneo pulmonar elevado. Se realizaron mediciones de espirometría antes y después de la transfusión de sangre en 18 nińos, con edad media de 14,2 (6.6-18.5) años. El aumento del volumen de sangre capilar pulmonar puede explicar, al menos parcialmente, las alteraciones de la función pulmonar en estos niños.

Para determinar los índices de la función pulmonar de los niños con anemia de células falciformes (ACF) que asisten a la clínica de falcemia pediátrica en el Hospital Universitario de Nigeria, Achigbu et al. ${ }^{6}$ evaluaron mediciones de la tasa de flujo espiratorio máximo, la capacidad vital forzada (FVC) y el volumen espiratorio forzado en el primer segundo (FEV1). Participó un total de 80 pacientes, que comprende $40 \mathrm{HbSS}$ homocigotos (hemoglobina SS) y un número igual de controles. Los índices de la función pulmonar fueron significativamente inferiores en los niños y adolescentes con ACF, en comparación con los controles emparejados con un genotipo AA hemoglobina.

Lunt et al. ${ }^{7}$ reclutaron dos grupos de nińos con edades entre 2 y 8 años, con un seguimiento entre 2 y 10 años. En ambos grupos de niños la función pulmonar se redujo significativamente, los episodios fueron más frecuentes durante el período de las crisis vasooclusivas. La función pulmonar se deterioró con el aumento de la edad en niños y la tasa de disminución fue mayor en los niños más pequeños, en los cuales los acontecimientos de Síndrome Torácico Agudo eran más comunes.

En otro estudio, con el objetivo de evaluar la función ventilatoria, mediante espirometría, en escolares y adolescentes con anemia falciforme, relacionando los hallazgos a parámetros clínicos y hematológicos, fueron evaluados portadores de falcemia de ambos géneros, a partir de los 10 años, clínicamente estables, fuera de complicaciones 


\section{Función pulmonar en falcémicos homocigotos de 5 a 15 años en consulta de hematología, Hospital Materno Infantil San Lorenzo de Los Mina agosto-octubre, 2015}

agudas. Se le sometió a la espirometría y se verificó la saturación transcutánea de oxígeno, los niveles de hemoglobina y el recuento de leucocitos, así como la asociación de alteraciones a la espirometría con las características demográficas, clínicas y de laboratorio de los pacientes analizados.

Fueron estudiados 51 pacientes, y en 40 de ellos $(78,4 \%)$ se identificó compromiso del perfil espirométrico, de los que: $20(50 \%)$ presentaron alteración ventilatoria mixta o combinada; 13 (32,5\%) tuvieron perfil restrictivo clásico; y siete $(17,5 \%)$ alteración ventilatoria obstructiva. De los siete, en cinco $(71,4 \%)$ se observó respuesta broncodilatadora positiva. El recuento total de leucocitos se asoció a la función pulmonar alterada. El volumen espiratorio forzado en el primer segundo sin broncodilatador, la capacidad vital forzada antes y después del broncodilatador y el flujo espiratorio forzado entre 25 y $75 \%$ de la capacidad vital forzada después del broncodilatador fue significativamente menor ${ }^{8}$.

El instituto de hematología e inmunología de la Habana, Cuba realizó un estudio descriptivo prospectivo, de septiembre de 1999 a septiembre de 2000, en donde se evaluaron las modificaciones funcionales ventilatorias en pacientes con anemia drepanocítica y antecedentes de Síndrome Torácico Agudo, siendo el trastorno restrictivo el más frecuentemente encontrado?

Rivera ${ }^{10}$ realizó un estudio retrospectivo en el Hospital Materno Infantil San Lorenzo de Los Mina, República Dominicana, con el objetivo de determinar la frecuencia de internamientos por neumonía en nińos falcémicos homocigotos menores de 15 años que han recibido la vacuna antineumocócica conjugada, en el período 2012-2015. De 104 niños falcémicos homocigotos que recibieron la vacuna antineumocócica conjugada, 48 (46.1\%) niños fueron ingresados con diagnóstico de neumonía; el 45.9\% de los pacientes era de 5-9 años; el $31.2 \%$ presentó neumonía a repetición; mientras que el $16.7 \%$ presentó asma.
González de la Rosa ${ }^{11}$ realizó un estudio para determinar la frecuencia de neumonía en pacientes falcémicos durante el período 2009-2013, en el Hospital Materno Infantil San Lorenzo de los Mina, República Dominicana. Este estudio reveló que el $98.8 \%$ de los pacientes tenía neumonía como causa de internamiento y/o de alguna complicación.

Almonte Guzmán et al. ${ }^{12}$ realizaron un estudio en el Hospital Materno Infantil San Lorenzo de los Mina, República Dominicana, con el objetivo de determinar las causas de ingreso más frecuentes en niños de 0 meses a 15 años con anemia falciforme, ingresados en el período enero-diciembre de 2013. Fue un estudio descriptivo retrospectivo de corte transversal. La causa de ingreso más frecuente fue la neumonía, con un $44 \%$.

\section{Material y métodos}

Se realizó un estudio descriptivo, retrospectivo, para determinar la función pulmonar en pacientes falcémicos homocigotos de 5 a 15 años, en consulta de hematología en el Hospital Materno Infantil San Lorenzo de Los Mina, período agosto-octubre de 2015. La población estuvo constituida por los expedientes de los pacientes durante el período de estudio, para un total de 200 pacientes; la muestra estuvo comprendida por un total de 23 expedientes de pacientes que tenían realizadas pruebas de función pulmonar en el momento del estudio. Se incluyó todo paciente homocigoto, entre 5 y 15 ańos de edad, que tuviera espirometría realizada; se excluyeron todos aquellos pacientes cuyos expedientes estuvieran incompletos o sin espirometría en el momento del estudio.

\section{Resultados}

La edad más afectada corresponde al grupo de 10-15 ańos (57\%). Los resultados fueron muy similares según el sexo: $48 \%$ femeninas y $52 \%$ masculinos (ver cuadros 1 y 2 ).

La neumonía fue la enfermedad pulmonar previa más común en los casos afectados $(48 \%)$ y el 
Síndrome Torácico Agudo (26\%) fue la principal complicación (ver cuadros 3 y 4).

Todos habían recibido la vacuna antineumocócica y el 74\% había recibido transfusión sanguínea (ver cuadros 5 y 6 ).

La terapia con hidroxiurea es la más utilizada (ver cuadro 7) y los niveles de hemoglobina más bajos (3-6 gr/Dl) se reportaron en el $48 \%$ de los pacientes (ver cuadro 8).

El patrón ventilatorio restrictivo aparece en un $78 \%$ (ver cuadro $9 \mathrm{~A}$ ) y su relación con la terapia empleada se expresa en el cuadro $9 \mathrm{~B}$.

Cuadro 1. Según edad

\begin{tabular}{lcc}
\hline Edad & $\begin{array}{c}\text { No. De casos } \\
\text { (n) }\end{array}$ & $\%$ \\
\hline $5-9^{\mathrm{a}}$ & 10 & 43 \\
$10-15^{\mathrm{a}}$ & 13 & 57 \\
Total & 23 & 100 \\
\hline
\end{tabular}

Cuadro 2. Según sexo

\begin{tabular}{lcc}
\hline Sexo & $\begin{array}{c}\text { No. De casos } \\
\text { (n) }\end{array}$ & $\%$ \\
\hline Femenina & 11 & 48 \\
Masculino & 12 & 52 \\
Total & 23 & 100 \\
\hline
\end{tabular}

Cuadro 3. Según enfermedad pulmonar previa

\begin{tabular}{lcc}
\hline Complicaciones & $\begin{array}{c}\text { No. De Casos } \\
\text { (n) }\end{array}$ & $\%$ \\
\hline Síndrome Torácico Agudo & 6 & 26 \\
Accidente cerebro & 1 & 4 \\
vascular & & \\
Ninguno & 16 & 70 \\
Total & 23 & 100 \\
\hline
\end{tabular}

Cuadro 4. Según complicaciones

\begin{tabular}{lcc}
\hline Enfermedad pulmonar previa & $\begin{array}{c}\text { No. Casos } \\
\text { (n) }\end{array}$ & $\%$ \\
\hline Neumonía & 11 & 48 \\
Asma & 6 & 26 \\
Asma +Neumonía & 6 & 26 \\
Total & 23 & 100 \\
\hline
\end{tabular}

Cuadro 5. Según vacuna antineumocócica

\begin{tabular}{lcc}
\hline Vacuna antineumocócica & $\begin{array}{c}\text { No. De casos } \\
\text { (n) }\end{array}$ & $\%$ \\
\hline $\mathrm{Si}$ & 23 & 100 \\
$\mathrm{~N} 0$ & 0 & 0 \\
Total & 23 & 100 \\
\hline
\end{tabular}

\section{Cuadro 6. Según transfusión sanguínea}

\begin{tabular}{lcc}
\hline Transfusión sanguínea & $\begin{array}{c}\text { No. De casos } \\
\text { (n) }\end{array}$ & $\%$ \\
\hline $\mathrm{Si}$ & 17 & 74 \\
$\mathrm{No}$ & 6 & 26 \\
Total & 23 & 100 \\
\hline
\end{tabular}

Cuadro 7. Según tratamiento de sostén

\begin{tabular}{lcc}
\hline Tratamientos de sostén & $\begin{array}{c}\text { No. De ca- } \\
\text { sos } \\
\text { (n) }\end{array}$ & $\%$ \\
\hline Hidroxiurea & 16 & 70 \\
Ninguna & 4 & 17 \\
Hidroxiurea+ terapia transfusio- & 3 & 13 \\
nal & & \\
Total & 23 & 100 \\
\hline
\end{tabular}


Función pulmonar en falcémicos homocigotos de 5 a 15 años en consulta de hematología, Hospital Materno Infantil San Lorenzo de Los Mina agosto-octubre, 2015

\section{Cuadro 8. Según niveles de hemoglobina y hematócrito}

\begin{tabular}{cccc}
\hline \multicolumn{4}{c}{ Niveles de hemoglobina y hematócrito } \\
\hline Hemoglobina g/dl & Hematócrito \% & $\begin{array}{c}\text { No. De casos } \\
\text { (n) }\end{array}$ & $\%$ \\
\hline 12 o más & $35-37$ & 5 & 22 \\
$10-11$ & $30-34$ & 4 & 17 \\
$7-9$ & $25-29$ & 3 & 13 \\
$3-6$ & $18-24$ & 11 & 48 \\
Total & & 23 & 100 \\
\hline
\end{tabular}

Cuadro 9 A. Según patrón ventilatorio

\begin{tabular}{lcc}
\hline Patrón ventilatorio & $\begin{array}{c}\text { No. De casos } \\
\text { (n) }\end{array}$ & $\%$ \\
\hline Normal & 4 & 18 \\
Obstructivo & 1 & 4 \\
Restrictivo & 18 & 78 \\
Total & 23 & 100 \\
\hline
\end{tabular}

Cuadro 9 B. Relación patrón ventilatorio/tratamiento de sostén

\begin{tabular}{|c|c|c|c|c|c|}
\hline \multirow[t]{2}{*}{$\begin{array}{l}\text { Patrón } \\
\text { ventilatorio/ } \\
\text { tratamiento } \\
\text { de sostén } \\
\text { Hidroxiurea }\end{array}$} & \multicolumn{2}{|c|}{$\begin{array}{l}\text { Normal } \\
\text { (n) } \%\end{array}$} & $\begin{array}{l}\text { Obstructivo } \\
\text { (n) } \%\end{array}$ & \multicolumn{2}{|c|}{$\begin{array}{c}\text { Restrictivo } \\
\text { (n) } \%\end{array}$} \\
\hline & 2 & 9 & & 13 & 57 \\
\hline Ninguna & 1 & 4 & 1 & 4 & 17 \\
\hline $\begin{array}{l}\text { Hidroxiurea+ } \\
\text { terapia } \\
\text { transfusional }\end{array}$ & 1 & 4 & & 1 & 4 \\
\hline Total & & 4 & 1 & & 18 \\
\hline
\end{tabular}

\section{Discusión}

En el presente estudio sobre la función pulmonar en pacientes falcémicos, según la edad, el mayor número de casos estuvo representado por los pacientes de 10-15 años, equivalente a un 57\%, lo que coincide con el estudio de Lunt et al. ${ }^{7}$, según el cual la edad más frecuente fue de 6.6 a 18.5 años.

La mayoría de nuestros pacientes evaluados no había presentado complicaciones, pero cabe destacar que, dentro de las que se encontraron, la más frecuente fue el Síndrome Torácico Agudo, con seis casos, lo que concuerda con un estudio realizado en Nigeria por Achigbu et al. ${ }^{6}$, sobre la función pulmonar en nińos con falcemia, en donde se reportó que los índices de la función pulmonar fueron significativamente inferiores en los niños y adolescentes con Síndrome Torácico Agudo.

Por otra parte, en un estudio realizado en Cuba por Guerra Paredes et al. ${ }^{13}$, en 2005, no se observa correlación entre la frecuencia de Síndrome Torácico Agudo y la disfunción ventilatoria detectada en los pacientes estudiados; esto nos indica que la repercusión ventilatoria en los pacientes con anemia drepanocítica debe ser tomada en cuenta aun en ausencia de traducción clínica, e independientemente del comportamiento clínico-hematológico de la enfermedad y de las complicaciones pulmonares presentes.

Todos nuestros pacientes habían recibido la vacuna antineumocócica, hecho que es de gran impacto 


\section{Claritza Elizabeth Concepción García, Rosa Nieves Paulino,}

Elsa Camilo Pantaleón y Rubén Darío Pimentel

en la disminución de la morbimortalidad por neumonía, que es la causa más común de ingreso hospitalario ${ }^{12}$. Gorrotxategi et al. ${ }^{14}$ señalan que las infecciones neumocócicas son una de las causas más importantes de morbilidad, hospitalización y mortalidad en todo el mundo, resultando más grave en niños menores de 2 años de edad y aún más frecuente en niños con antecedentes de enfermedades crónicas, tales como la falcemia.

La mayoría de los pacientes había recibido transfusión sanguínea, al igual que lo reportado por Rivera Fune $^{10}$, donde el $52.0 \%$ de los pacientes recibió transfusiones sanguíneas. Este hecho no es relevante, puesto que los pacientes con anemia falciforme reciben múltiples transfusiones sanguíneas como parte de su terapia de sostén.

El tratamiento de sostén con la hidroxiurea resultó alentador, tomando en cuenta que este es un medicamento que disminuye las complicaciones más frecuentes y el número de hospitalizaciones, y sería de gran importancia la realización de estudios que relacionen su uso con la función pulmonar y las diferentes complicaciones.

En cuanto a los niveles de hemoglobina y hematócrito, el mayor porcentaje correspondió a la escala de 3-6 g/dl y de 18-27\%, respectivamente, hallazgos que se asemejan a los reportados ${ }^{10}$.

El patrón espirométrico que predominó en este estudio fue el trastorno restrictivo, dato que coincide con un estudio realizado en 2010 por Mesa Cuervo et al. ${ }^{9}$, en Cuba, quienes concluyeron que la disfunción ventilatoria restrictiva se observó en la mayoría de los pacientes con falcemia, independientemente del antecedente de STA. Según una revisión de la literatura sobre alteraciones espirométricas en niños y adolescentes con anemia falciforme ${ }^{8}$, hay autores que plantean que los trastornos ventilatorios restrictivos pueden ser ocasionados por estrés patológico, que afecta ya sea la característica de distensibilidad de los pulmones o la pared torácica, que incluye el volumen máximo que es capaz de contener, o las fuerzas de los músculos respiratorios. $82 \%$ de los pacientes revelaron cambios en la función pulmonar tanto mixto como restrictivo; por lo que sus resultados indican que el patrón restrictivo es un hallazgo frecuente en esta enfermedad.

Además, plantean que la presencia del patrón restrictivo en esta población podría ser el resultado de episodios de vaso-oclusión en el pulmón, que es un órgano muy propenso a sufrir de esta condición, debido a sus características anatómicas; también puede ser el resultado de la respiración superficial causada por dolor de pecho o por alteraciones estructurales de la cavidad torácica debido a infartos de huesos, osteoporosis u osteomalacia en las vértebras, y a la propia fisiopatología de la enfermedad falciforme, con los fenómenos subclínicos de vaso-oclusión, con daño de tejidos y órganos, de los cuales el pulmón es el más frecuentemente afectado. Estos resultados sugieren que el daño causado por la falcemia puede afectar significativamente la función pulmonar, independientemente de las complicaciones, de la enfermedad pulmonar previa y del tratamiento de sostén.

\section{Bibliografía}

1. Sociedad Española de Hematología Y Oncología Pediátricas. Guía de práctica clínica sobre enfermedad de células falciformes pediátrica. Disponible en: www.SEHOP.org

2. Sociedad Española de Hematología y Oncología Pediátricas SEHOP-2010. Guía de práctica clínica sobre enfermedad de células falciformes pediátrica. Disponible es: www. SEHOP.org.

3. Fauci A, Fauci A, Braunwald E, Kasper D, Hauser E, Longo D, Jameson L, Loscalzo J. Harrison Medicina Interna. 17 ${ }^{\mathrm{a}}$ ed. México: Mcgraw-HillInteramericana; 2009. 1562 p. 


\section{Función pulmonar en falcémicos homocigotos de 5 a 15 años en consulta de hematología, Hospital Materno Infantil San Lorenzo de Los Mina agosto-octubre, 2015}

4. Serjean G. Sickle Cell Disease. International Course by The Medical Virtual University Of Monaco.Mónaco:UniversitéMédicaleVirtuelle De Monaco; 2011.

5. Powars D. Sickle cell anemia and major organ failure. Hemoglobin. 1990; 14(6): 573-98.

6. Lunt A, McGhee E, Robinson P, Rees D, Height S, Greenough A. Lung function, transfusion, pulmonary capillary blood volume and sickle cell disease. Respir Physiol Neurobiol. 2016 feb. 1;222: 6-10. Disponible en: doi: 10.1016/j.resp.2015.11.006. Epub 2015 Nov 22

7. Achigbu KI, Odetunde OI, Chinawa JM, Achigbu EO, Ikefuna AN, Emodi IJ, Ibe BC. Pulmonary function indices in children with sickle cell anemia in Enugu, south-east Nigeria. Saudi Med J. 2015;36(8): 928-34.

8. Lunt A, McGhee E, Sylvester K, Rafferty G, Dick M, Rees D, Height S, Thein SL, Greenough A. Longitudinal assessment of lung function in children with sickle cell disease. Pediatr Pulmonol.

2016; 51(7): 717-723.

9. Fonseca Cássia SV, Araújo Melo CA, Carvalho RM, Barreto Neto J, Araújo Jerónimo G, Cipolotti R. Función pulmonar en portadores de anemia falciforme. Revista Paulista de Pediatría. 2011; 29(1): 85-90.

10. Mesa CJ. Modificaciones funcionales ventilatorias en pacientes con anemia drepanocítica y antecedentes de Síndrome Torácico Agudo. Rev. Cubana de hematología, inmunología y hemoterapia. 2002; 18(1): 1-7.
11. Rivera Fune WX. Frecuencia de internamientos por neumonía de niños falcémicos homocigotos menores de 15 ańos que han recibido la vacuna antineumocócica conjugada en el Hospital Materno Infantil San Lorenzo de Los Mina, enero 2012-febrero, 2015 (tesis de postgrado para optar por el título de especialista en Pediatría). Santo Domingo: Universidad Autónoma de Santo Domingo; 2015.

12. González de la Rosa KY. Frecuencia de neumonía en pacientes falcémicos menores de 15 años atendidos en el Hospital Materno Infantil San Lorenzo de Los Mina, durante el período 2009-2013 (tesis de postgrado para optar por el título de especialista en Pediatría). Santo Domingo: Universidad Autónoma de Santo Domingo; 2014.

13. Almonte Guzmán $S$ et al. Causas de ingreso más frecuentes en niños con anemia falciforme de 0 meses a 15 ańos ingresados en el Hospital Materno Infantil San Lorenzo de Los Mina en el período enero-diciembre 2013 (tesis de grado para optar por el título de doctor en Medicina). Santo Domingo: Universidad Autónoma de Santo Domingo; 2013.

14. Guerra Paredes M, Camacho Machín ML, Fortún Prieto A. Función pulmonar en pacientes con anemia drepanocítica. Revista de Ciencias Médicas 2005;9 (3) Pinar del Río julsep. 2005

15. Gorrotxategi Gorrotxategi P, Iturrioz Mata A. Vacuna conjugada contra el neumococo. ¿Es una prevención universal adecuada de la enfermedad neumocócica? Rev Pediatr Aten Primaria. 2010; 12: 1243-55 